\title{
Paraparesia aguda por hemangioma vertebral agresivo. Reporte de dos casos y revisión bibliográfica
}

\author{
Matías Pereira Duarte, Gastón Camino Wilhuber, Gonzalo Kido, Julio Bassani, Matías Petracchi, Carlos Solá, \\ Marcelo Gruenberg \\ Sector de Patología Espinal de Adulto, Servicio de Ortopedia y Traumatología, Hospital Italiano de Buenos Aires, \\ Ciudad Autónoma de Buenos Aires, Argentina
}

\section{RESUMEN}

Introducción: Los hemangiomas agresivos constituyen el 1\% del total de los hemangiomas vertebrales. Pueden producir dolor, fracturas, deformidad y compromiso neurológico, generalmente de larga evolución. Se han descrito diferentes opciones terapéuticas, pero el manejo óptimo sigue sin estar claro. Comunicamos dos casos de paraparesia aguda secundaria a un hemangioma torácico agresivo, describimos su tratamiento y evolución. Realizamos una revisión narrativa de la bibliografía. Conclusión: Mediante la descompresión y la artrodesis tempranas seguidas de radioterapia, se logró la recuperación neurológica completa y el control de la enfermedad en un seguimiento a mediano plazo.

Palabras clave: Hemangioma agresivo; tumor benigno; columna vertebral; radioterapia; paraparesia.

Nivel de Evidencia: IV

\section{Acute Paraparesis due to Aggressive Vertebral Hemangioma. Report of Two Cases and Literature Review}

\section{ABSTRACT}

Introduction: Aggressive hemangiomas make up 1\% of all vertebral hemangiomas. They can produce pain, fractures, deformity and slowly progressive neurological compromise. Different treatment approaches have been described, but optimal management remains unclear. We report two cases of acute paraparesis secondary to aggressive thoracic hemangioma, we describe their treatment and evolution. We carry out a narrative review of the literature on vertebral hemangiomas. Conclusion: Through early decompression and arthrodesis followed by local adjuvant radiotherapy, we obtained complete neurological recovery and disease control in a medium-term follow-up.

Keywords: Aggressive hemangioma; benign tumor; spine; radiotherapy; paraparesis.

Level of Evidence: IV

\section{INTRODUCCIÓN}

Los hemangiomas vertebrales (HV) son los tumores benignos diagnosticados con más frecuencia en el esqueleto axial, tienen una incidencia del 10-12\% entre la cuarta y quinta décadas de vida. ${ }^{1,2}$ Histológicamente están constituidos por espacios vasculares revestidos con endotelio separados por tabiques de estroma óseo. ${ }^{3,4}$ En general, son asintomáticos y quiescentes, la mayoría se descubre de manera incidental al realizar una resonancia magnética (RM) indicada por otros motivos. Afectan principalmente el cuerpo vertebral y, rara vez, se extienden a los componentes posteriores. ${ }^{5}$

En los estudios por imágenes, los HV “típicos” exhiben un patrón característico, focos líticos con trabéculas que recuerdan un panal de abejas con grandes y largas estrías verticales en la radiografía simple. En la tomografía computarizada, se observan calcificaciones puntiformes irregularmente distribuidas, producto de la coalescencia de las trabéculas verticales descritas. La RM destaca una imagen hiperintensa tanto en secuencia T1 como en T2 (debido al predominio del contenido graso), así como también en secuencias sensibles a los líquidos, STIR, debido a los

Recibido el 19-7-2020. Aceptado luego de la evaluación el 2-9-2020 • Dr. MATíAS PEREIRA DUARTE • matiaspereiraduarte@ gmail.com

Cómo citar este artículo: Pereira Duarte M, Camino Willhuber G, Kido G, Bassani J, Petracchi M, Solá C, Gruenberg M. Paraparesia aguda por hemangioma vertebral agresivo. Reporte de dos casos y revisión bibliográfica. Rev Asoc Argent Ortop Traumatol 2021;86(3):398-406. https://doi.org/10.15417/issn.1852-7434.2021.86.3.1161 
componentes vasculares. ${ }^{5-7}$ Sin embargo, los HV pueden no mostrar estas características distintivas cuando hay un menor contenido graso y más contenido vascular, son los llamados HV "atípicos". ${ }^{5}$

Los HV "agresivos" son la tercera categoría de estas lesiones que, aunque son benignos desde el punto de vista histológico, tienen una conducta localmente más agresiva, corresponden al grado 3 de la clasificación de Enneking. ${ }^{8}$ Son poco frecuentes, representan el $1 \%$ de todos los HV, ${ }^{9}$ y pueden provocar síntomas, como dolor en el 54\% de los casos, fracturas patológicas, deformidad de la columna y compromiso neurológico hasta en un $40 \%{ }^{10-13} \mathrm{Se}$ localizan más frecuentemente en la columna dorsal y la relación hombre:mujer es 1:1,5. ${ }^{14}$

El objetivo de este artículo es presentar dos casos de paraparesia aguda debida a hemangiomas torácicos agresivos y realizar una revisión narrativa de la bibliografía sobre el tema.

\section{CASO CLÍNICO 1}

Hombre de 22 años que consultó por pérdida progresiva de la fuerza motora en los miembros inferiores asociada a dorsalgia secundaria a una caída desde su propia altura, siete días antes. En el examen físico, presentaba una fuerza muscular 2/5 para los grupos musculares inervados por L2, L3, L4, L5 y S1, hipoestesia por debajo del área umbilical, y reflejos rotulianos y aquilianos hiperreactivos. El puntaje de la escala de Nurick ${ }^{15}$ fue 5 y el de la escala modificada de la Japanese Orthopaedic Association (mJOA) ${ }^{16}$ resultó de 9/18. Se diagnosticó un síndrome medular incompleto con una paraparesia severa, Frankel C.

Las radiografías mostraron una alteración de la trama ósea en el cuerpo vertebral de T9 (Figura 1). Se realizó una tomografía computarizada en la cual no se observó el patrón clásico puntiforme de los hemangiomas, lo cual dificultó el diagnóstico inicial. La RM mostró una imagen isointensa en las secuencias T1 y T2 con extensión extravertebral y compromiso del canal medular, mientras que, en la secuencia STIR, la lesión era francamente hiperintensa (Figura 2).

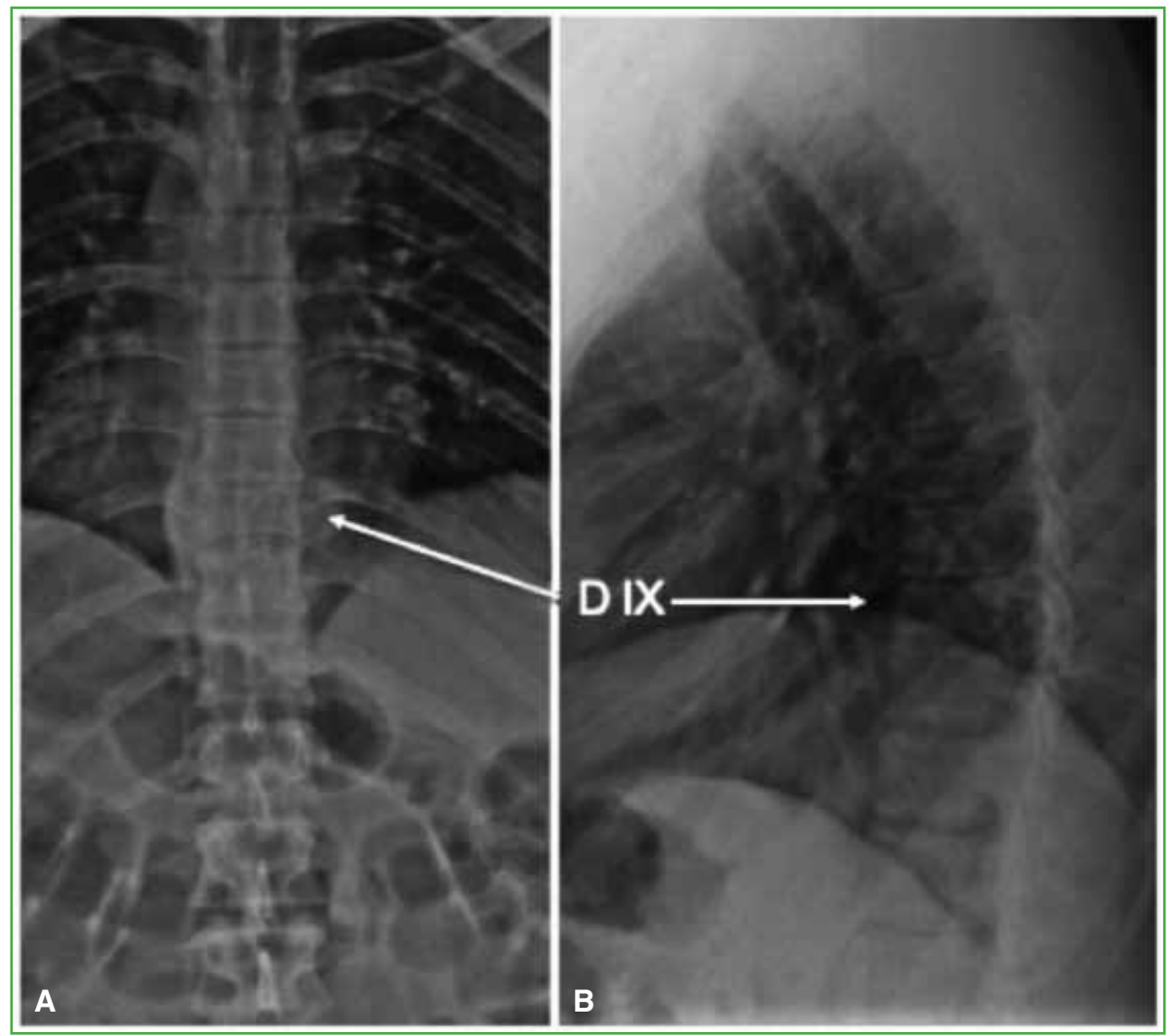

Figura 1. Caso 1. Hombre de 22 años. Radiografías anteroposterior (A) y lateral (B) de columna dorsal. Se observa una alteración en la trama ósea a nivel de T9 y un colapso leve del cuerpo vertebral. 


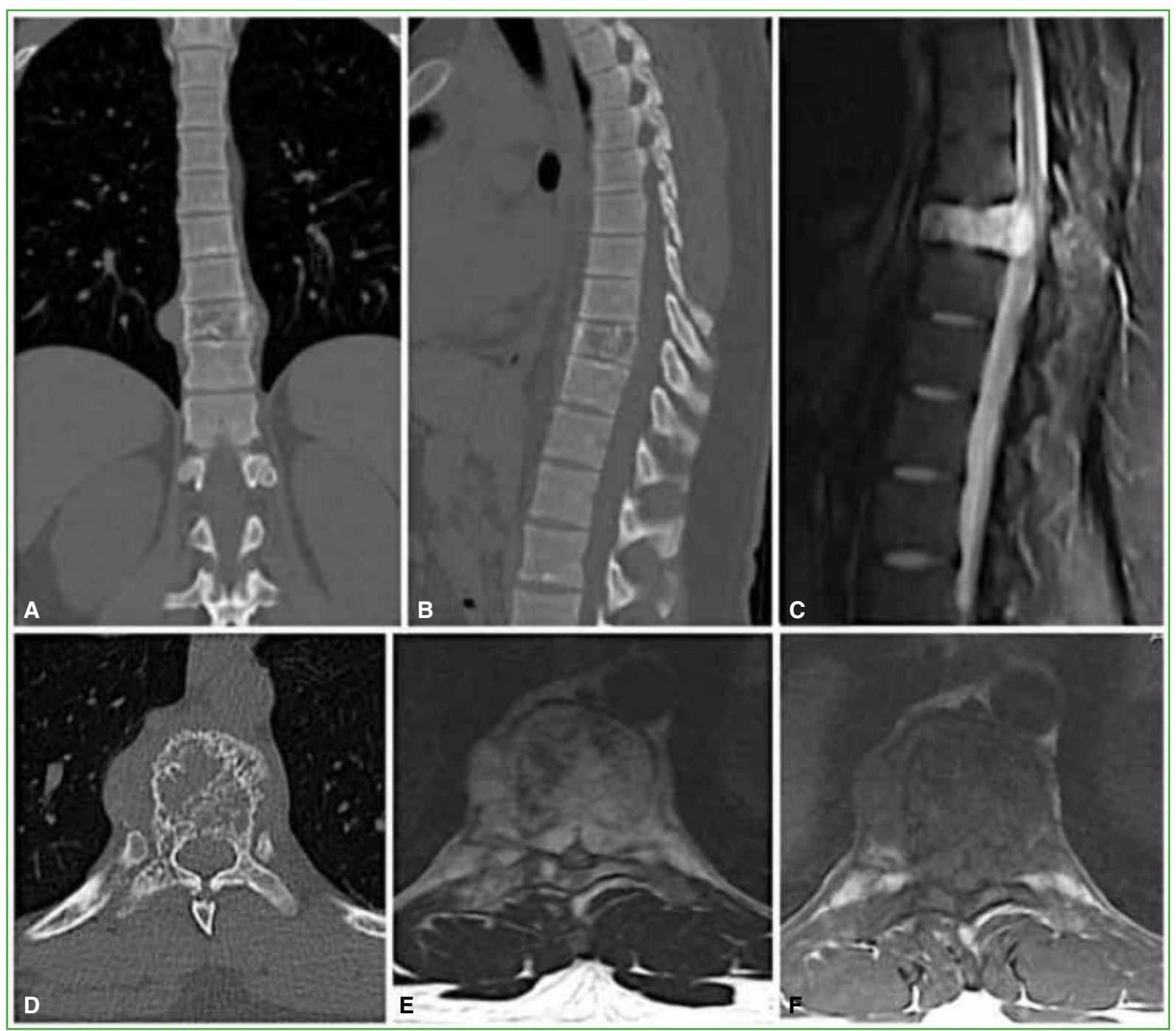

Figura 2. Caso 1. Hombre de 22 años. Tomografía computarizada de columna toracolumbar. A. Corte coronal.

B. Corte sagital, D. Corte axial. Se observan lesiones líticas, heterogéneas con un patrón permeativo, que alteran la trama ósea del cuerpo vertebral de T9 con compromiso de las corticales y extendiéndose a espacios paravertebrales y epidural. Se evidencia el compromiso de ambos pedículos a predominio derecho, con afectación de la pars y facetas homolaterales. Resonancia magnética de columna dorsal. C. Corte sagital, secuencia STIR. Se destaca la hiperintensidad de la lesión que afecta a todo el cuerpo vertebral, generando una fractura patológica, con compromiso del muro posterior y el canal medular. E. Secuencia T1. F. Secuencia T2. Imágenes de carácter isointenso, se demuestra una mayor extensión paravertebral derecha.

Debido al cuadro neurológico agudo y progresivo, se realizó una descompresión mediante laminectomía de T9 y una artrodesis de T7-T11 de urgencia por vía posterior (Figura 3). Se enviaron muestras para el análisis histopatológico de la lesión, que informó el diagnóstico de HV (Figura 4).

A las cinco semanas de la cirugía, el paciente comenzó el tratamiento radiante con una dosis total de 4000 cGy fraccionados en sesiones de 150-200 cGy, cada 24-48 h, según la tolerancia, con el objetivo de prevenir las recurrencias.

A los 36 meses de la intervención, luego de una rehabilitación progresiva intensa, el paciente presenta una mejoría neurológica, con puntajes de 17/18 en la escala mJOA, Nurick 3 y Frankel E, sin signos de recurrencia tumoral. 


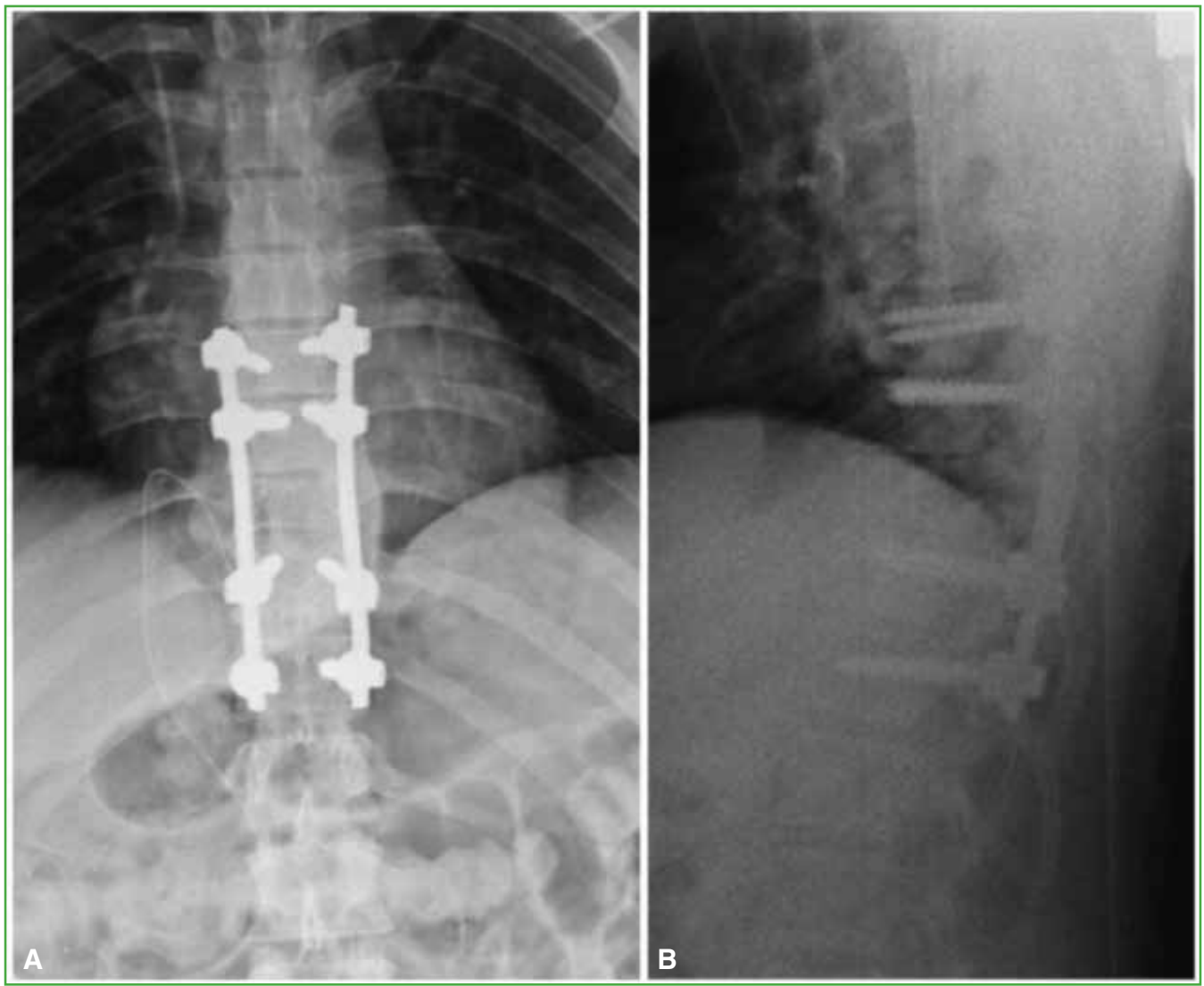

Figura 3. Caso 1. Hombre de 22 años. Radiografías anteroposterior (A) y lateral (B) de columna dorsal, posoperatorias. Se visualiza la fijación con tornillos pediculares de T7 a T11.

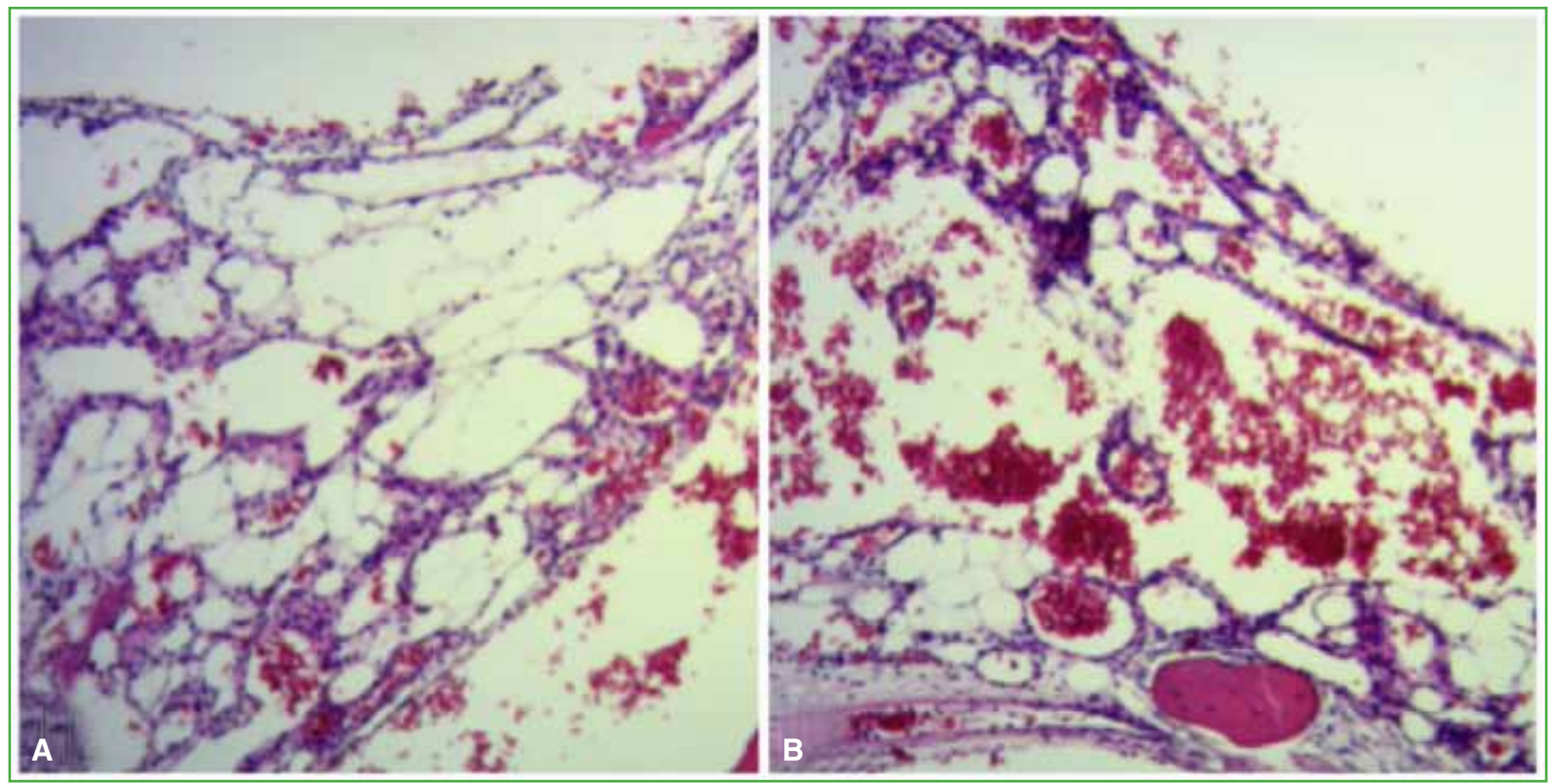

Figura 4. Caso 1. Hombre de 22 años. El análisis histológico bajo microscopia x20 (A) y x40 (B) y tinción con hematoxilina-eosina mostró el revestimiento de los espacios creados dentro del estroma de matriz colágena por delgadas capas de células endoteliales planas que forman vasos sanguíneos cavernosos de tamaño capilar, compatible con el diagnóstico de hemangioma. 


\section{CASO CLÍNICO 2}

Mujer de 23 años que consulta en la central de emergencias por dorsalgia progresiva y paraparesia de $24 \mathrm{~h}$ de evolución, sin antecedente traumático de relevancia. Como antecedente de jerarquía, la paciente refirió episodios previos de dorsalgia con estudios por imágenes y punción biopsia vertebral compatible con $\mathrm{HV}$, tratado de manera conservadora. En el examen físico, presentaba una fuerza muscular disminuida 3/5 desde L2 hacia distal, hiperreflexia rotuliana bilateral y Babinski positivo. Los puntajes neurológicos eran de 4/5 para Nurik, 14/18 para la escala mJOA y Frankel C. La RM de urgencia reveló el compromiso completo de T11, con alteración del entramado óseo, extensión a través del pedículo derecho hacia el anillo apofisario posterior e invasión del canal medular (Figura 5). Ante el diagnóstico previo de HV, se realizó una embolización selectiva de la arteria nutricia de la lesión, seguida de una descompresión de T10-T12 y artrodesis de T9-L1 por vía posterior (Figura 6). A las cuatro semanas de la cirugía, comenzó con el tratamiento radiante a dosis de 4500 cGy. Luego de 60 meses de seguimiento, la paciente no tiene dolor, y sus puntajes son Frankel E, Nurik 1 y 17/18 en la escala mJOA. No se observaron signos de recurrencia de la lesión.
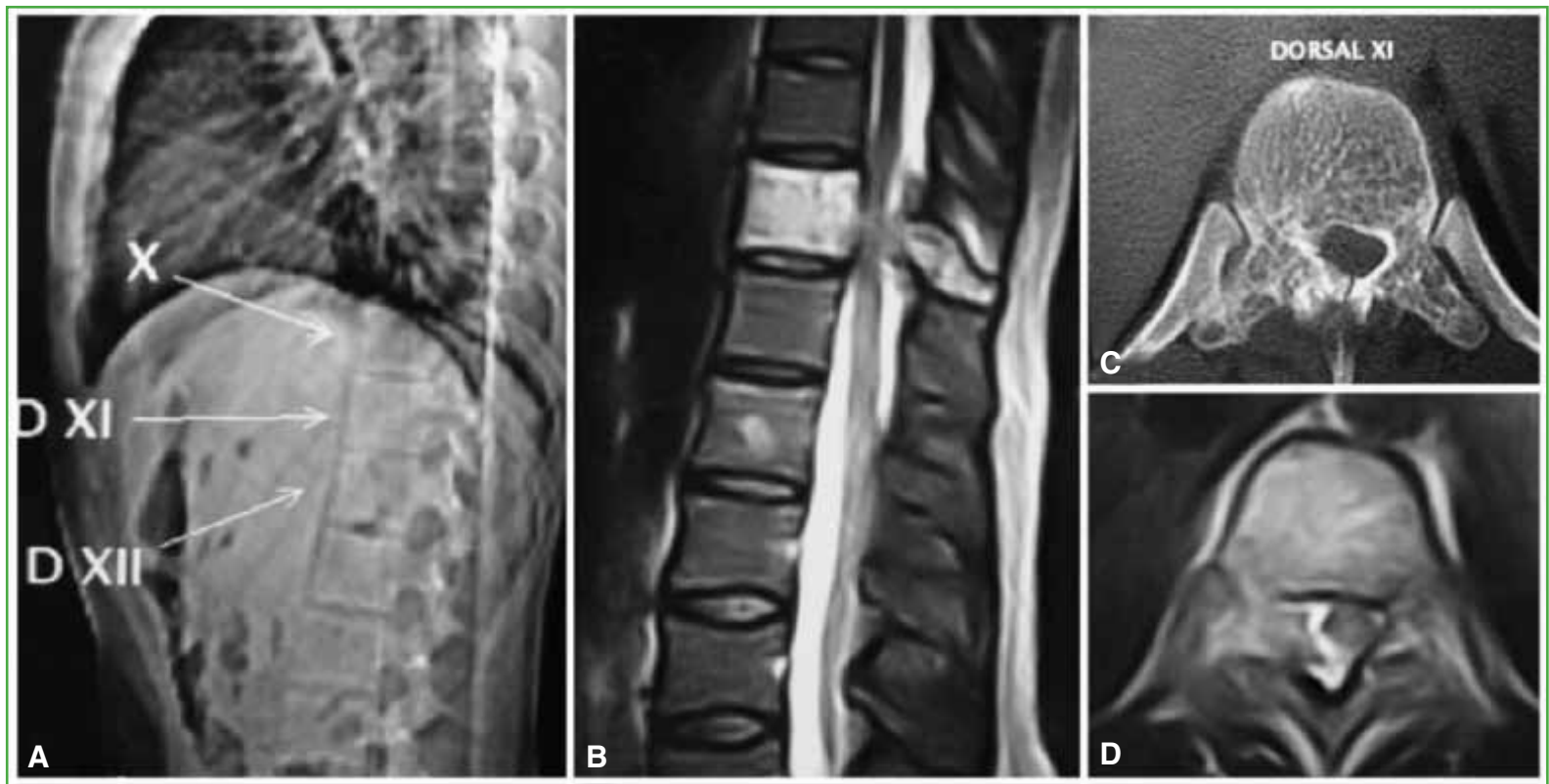

Figura 5. Caso 2. Mujer de 23 años. A. Radiografía lateral de columna dorsal con marcación del nivel involucrado sin hallazgos de relevancia. Resonancia magnética. B. Corte sagital, secuencia T2. Se observa hiperintensidad del cuerpo vertebral con extensión hacia el pedículo derecho e invasión del canal medular, desplazando la médula hacia la izquierda (D). C. Tomografía computarizada, corte axial. Se visualiza la alteración de la trama ósea en el cuerpo vertebral, con insuflación de los componentes derechos del anillo apofisario. 

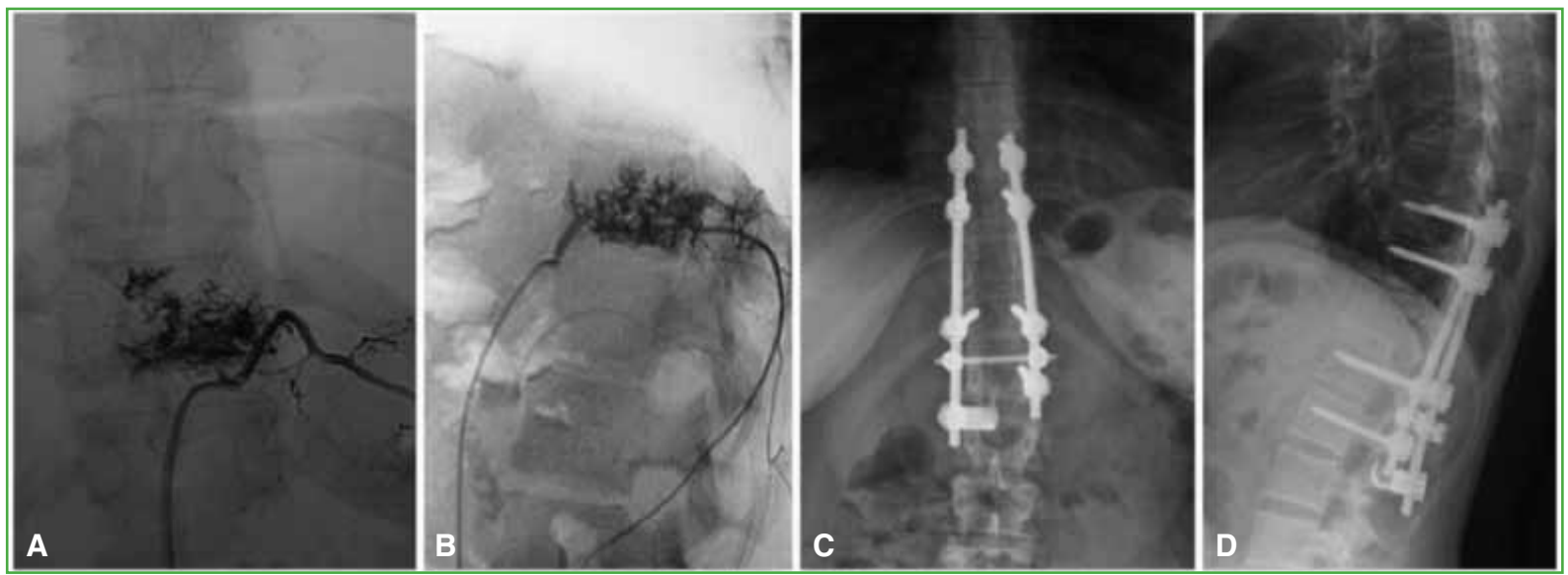

Figura 6. Caso 2. Mujer de 23 años. Imágenes fluoroscópicas anteroposterior (A) y lateral (B) de la lesión de la columna dorsal a nivel de T11 durante el procedimiento de embolización arterial selectiva de la arteria nutricia. Radiografías anteroposterior (C) y lateral (D) de columna dorsal a los 5 años de seguimiento. Se visualizan la descompresión y la artrodesis dorsolumbar de T9 a L1.

\section{DISCUSIÓN}

Describimos dos casos de hemangioma agresivo dorsal con paraparesia aguda grave que requirieron resolución quirúrgica de urgencia. Los HV agresivos representan menos del $1 \%$ del total, pueden producir dolor, fracturas patológicas y compromiso neurológico. La mayoría de los hemangiomas agresivos exhiben un patrón típico "a lunares" (polka-dot sign) ${ }^{17}$ o en panal de abejas en las imágenes; sin embargo, hasta en un tercio, los patrones pueden ser atípicos. ${ }^{18}$ Acosta y cols. ${ }^{19}$ describen seis particularidades en los estudios por imágenes, que se asocian a HV "sintomáticos" o "agresivos": 1) ubicación torácica (particularmente T3-9), 2) afectación de todo el cuerpo vertebral, 3) afectación del arco neural (particularmente los pedículos), 4) una apariencia irregular sin forma de panal, 5) una corteza expandida, insuflada y mal definida y 6) afección del tejido blando paravertebral adyacente. El diagnóstico por imágenes de HV “atípicos” y "agresivos" puede ser difícil, independientemente de la modalidad de imagen utilizada, ya que pueden imitar tumores malignos óseos o metástasis primarias. ${ }^{5}$ Nuestros dos pacientes presentaban todas estas características consideradas atípicas, lo que dificultó el diagnóstico por las imágenes y fue preciso recurrir a la anatomía patológica de la lesión para un diagnóstico de certeza.

Las estrategias de tratamiento óptimas y las opciones de terapia adyuvante siguen siendo controvertidas, ya que es difícil llevar a cabo grandes estudios aleatorizados debido a la baja prevalencia de este cuadro. ${ }^{1,19,20}$ Se han descrito diferentes opciones, como la inyección intralesional de etanol, ${ }^{21}$ la embolización arterial, ${ }^{22}$ la vertebroplastia ${ }^{23}$ o cifoplastia, ${ }^{24}$ la resección radical total (espondilectomía) ${ }^{19}$ o parcial más estabilización, ${ }^{25,26}$ la radioterapia ${ }^{27,28} \mathrm{o}$ una combinación de ellas. Recientemente Kaoudi y cols. ${ }^{29}$ describieron la ablación térmica por radiofrecuencia asistida por robot en un hemangioma agresivo del sacro, con buenos resultados al año de seguimiento.

La embolización intrarterial preoperatoria ofrece como ventajas el control de la hemorragia y la reducción de la morbilidad perioperatoria. ${ }^{30}$ En algunos casos, se ha observado el enlentecimiento o la detención del crecimiento de la lesión cuando se realiza de manera aislada. ${ }^{3,31}$ En el primer caso presentado, no se consideró la embolización debido a la falta de diagnóstico específico y a la urgencia del cuadro. Sin embargo, en el segundo caso, se contaba con la confirmación histológica previa, por lo que se pudo realizar una embolización de urgencia seguida de la cirugía de descompresión y la artrodesis.

El uso de radioterapia adyuvante después de las cirugías de escisión intralesional con el objetivo de disminuir la tasa de recurrencia es controvertido. Mientras algunos autores han comunicado buenos resultados a largo plazo en series de pacientes tratados de esta manera, ${ }^{1,11,32}$ Qiu y cols. ${ }^{20}$ informaron haber realizado radioterapia solo en tres de 10 pacientes, y tampoco detectaron recurrencias a los 11 meses de seguimiento promedio, por lo que se pone en duda el real beneficio de este tratamiento. 
Ambos pacientes presentados recibieron radioterapia a la cuarta y quinta semana después de la cirugía; esta demora permite disminuir la tasa de complicaciones de la herida, como la dehiscencia y la infección. No se detectaron complicaciones posoperatorias y ambos pacientes tuvieron una recuperación neurológica progresiva y completa sin signos de recidiva tumoral al final del seguimiento.

Describimos dos casos de una patología rara con una presentación infrecuente. Los reportes sobre síndromes medulares agudos debidos a hemangiomas agresivos son escasos, la mayoría se trata de cuadros de lenta progresión en el tiempo. En un análisis retrospectivo, Qiu y cols. ${ }^{20}$ comunican 10 casos de hemangiomas agresivos en un período de 13 años, cinco de ellos con un deterioro neurológico progresivo y un Frankel D al momento del tratamiento. Nuestros pacientes se presentaron con un Frankel $\mathrm{C}$ desarrollado en un corto período. Obtuvimos buenos resultados luego de las cirugías de urgencia con descompresión y estabilización seguidas de tratamiento radiante. Es importante destacar que el éxito de este tipo de cirugías depende de la rapidez en la detección, el diagnóstico y el tratamiento, ya que la demora puede provocar una mejoría neurológica menos marcada o ausente.

Se ha publicado una tasa de recurrencia posquirúrgica del $0 \%$ al $30 \%,{ }^{20}$ al margen del tratamiento con radioterapia adyuvante o sin ella. Cabe destacar la particular agresividad de esta lesión en embarazadas, en quienes la tasa de recurrencia es superior al $20 \%{ }^{19,33}$ Esto es producto de la influencia de la progesterona gestacional sobre el tumor, lo cual también se relacionaría con la mayor incidencia en mujeres. ${ }^{14}$

Según nuestra búsqueda, estos son los dos primeros casos publicados en la bibliografía latinoamericana sobre compromiso neurológico agudo en pacientes jóvenes provocado por HV agresivos. Creemos que es importante conocer este diagnóstico, sus imágenes típicas y atípicas, así como sus formas de tratamiento para poder brindar a nuestros pacientes los mejores resultados. En la actualidad, no existe consenso sobre el tratamiento óptimo y cada caso debe ser evaluado particularmente teniendo en cuenta todos los factores enumerados antes.

\section{CONCLUSIÓN}

Presentamos dos casos de paraparesia aguda grave secundaria a HV torácico agresivo. Mediante la descompresión y la artrodesis tempranas seguidas de radioterapia, se logró la recuperación neurológica completa y el control de la enfermedad en un seguimiento a mediano plazo.

Conflicto de intereses: Los autores no declaran conflictos de intereses.

\section{BIBLIOGRAFÍA}

1. Fox MW, Onofrio BM. The natural history and management of symptomatic and asymptomatic vertebral hemangiomas. J Neurosurg 1993;(7881):36-45. https://doi.org/10.3171/jns.1993.78.1.0036

2. Huvos AG. Hemangioma, lymphangioma, angiomatosis/lymphangiomatosis, glomus tumor. En: Huvos AG (ed.). Bone tumors: diagnosis, treatment, and prognosis. $2^{\text {nd }}$ ed. Philadelphia: Saunders; 1991:553-78.

3. Rudnick J, Stern M. Symptomatic thoracic vertebral hemangioma: a case report and literature review. Arch Phys Med Rehabil 2004;85(9):1544-47. https://doi.org/10.1016/j.apmr.2003.08.099

4. Cross JJ, Antoun NM, Laing RJ, Xuereb J. Imaging of compressive vertebral haemangiomas. Eur Radiol 2000;10(6):997-1002. https://doi.org/10.1007/s003300051051

5. Gaudino S, Martucci M, Colantonio R, Lozupone E, Visconti E, Leone A, et al. A systematic approach to vertebral hemangioma. Skeletal Radiol 2015;44(1):25-36. https://doi.org/10.1007/s00256-014-2035-y

6. Orrison W. Neuroimaging. Philadelphia: W. B. Saunders Co; 2000:1335-6.

7. Osborn A. Diagnostic neuroradiology. St. Louis, MO: Mosby; 1994:877-9. 
8. Enneking WF. Musculoskeletal tumor staging: 1988 update. Cancer Treat Res 1989;44:39-49. https://doi.org/10.1007/978-1-4613-1757-9_3

9. Healy M, Rerz DA, Pearl L. Spinal hemangiomas. Neurosurgery 1985;13(6):689-91. https://doi.org/10.1227/00006123-198312000-00013

10. Templin CR, Stambough JB, Stambough JL. Acute spinal cord compression caused by vertebral hemangiomas. Spine J 2004;4(5):595-600. https://doi.org/10.1016/j.spinee.2003.08.034

11. Castel E, Lazennec JY, Chiras J, Enkaoua E, Saillant G. Acute spinal cord compression due to intraspinal bleeding from a vertebral hemangioma: two case-reports. Eur Spine J 1999;8(3):244-8. https://doi.org/:10.1007/s005860050167

12. Ahn H, Jhaveri S, Yee A, Finkelstein J. Lumbar vertebral hemangioma causing cauda equina syndrome: a case report. Spine (Phila Pa 1976) 2005;30(21):662-4. https://doi.org/10.1097/01.brs.0000184560.78192.f6

13. Nguyen JP, Djinjian M, Pavlovitch JM, Badiane S. Vertebral hemangioma with neurologic signs: therapeutic results. Neurochirurgie 1989;35(5):299-303, 305-8. PMID: 2630927

14. Campanacci M. Hemangioma. En: Campanacci M (ed.). Bone and soft tissue tumors. Padova: Piccin Nuova Libraria \&Wien: Springer; 1999:599-618.

15. Nurick S. The pathogenesis of the spinal cord disorder associated with cervical spondylosis. Brain 1972;95(1):87100. https://doi.org/10.1093/brain/95.1.87

16. Tetreault L, Kopjar B, Nouri A, Arnold P, Barbagallo G, Bartels R, et al. The modified Japanese Orthopaedic Association scale: establishing criteria for mild, moderate and severe impairment in patients with degenerative cervical myelopathy. Eur Spine J 2017;26(1):78-84. https://doi.org/10.1007/s00586-016-4660-8

17. Persaud T. The polka-dot sign. Radiology 2008;246(3):980-1. https://doi.org/10.1148/radiol.2463050903

18. Wang B, Zhang L, Yang S, Han S, Jiang L, Wei F, et al. Atypical radiographic features of aggressive vertebral hemangiomas. J Bone Joint Surg Am 2019;101(11):979-86. https://doi.org/10.2106/JBJS.18.00746

19. Acosta FL, Sanai N, Cloyd J, Deviren V, Chou D, Ames CP. Treatment of Enneking stage 3 aggressive vertebral hemangiomas with intralesional spondylectomy: report of 10 cases and review of the literature. $J$ Spinal Disord Tech 2011;24(4):268-75. https://doi.org/10.1097/BSD.0b013e3181efe0a4

20. Qiu B, Joo P, Ajabnoor R, Boyce B, Mesfin A. Surgical management of aggressive hemangiomas of the spine. J Clin Neurosci 2020;78:67-72. https://doi.org/10.1016/j.jocn.2020.06.012

21. Heiss JD, Doppman JL, Olfield EH. Brief report: relief of spinal cord compression from vertebral hemangioma by intralesional injection of absolute etanol. N Engl J Med 1994;331(8):508-11. https://doi.org/10.1056/NEJM199408253310804

22. Hekster REM, Luyendijk W, Tan TI. Spinal cord compression caused by vertebral hemangiomas relieved by percutaneous catheter embolisation. Neuroradiology 1972;3:160-4. https://doi.org/10.1007/BF00341502

23. Hao J, Hu Z. Percutaneous cement vertebroplasty in the treatment of symptomatic vertebral hemangiomas. Pain Physician 2012;15(1):43-9. PMID: 22270737

24. Pinto M, Añon M, D’Innocenzo AC, Gomez AA, Zanabria Sosa C, Gonzalez FJ. Cifoplastia con balón para el tratamiento de los hemangiomas vertebrales. Descripción de un caso y revisión bibliográfica. Rev Asoc Arg Ortop Traumatol 2009;74(1):66-9. Disponible en: https://www.aaot.org.ar/revista/2009/n1/art11.pdf

25. Garzon-Muvdi T, Iyer R, Goodwin CR, Abu-Bonsrah N, Orru E, Gailloud P, et al. Percutaneous embolization and spondylectomy of an aggressive L2 hemangioma. Spine J 2016;16(3):167-8. https://doi.org/10.1016/j.spinee.2015.10.001

26. Jaramillo Carling E. Compresión medular causada por hemangioma vertebral. Presentación de un caso y análisis de la literatura médica. Rev Colomb Ortop Traumatol 2005;19(1):86-9. Disponible en: http://www.sccot.org.co/pdf/RevistaDigital/19-01-2005/14Compresion.pdf

27. Sewell MD, Dbeis R, Bliss P, Watkinson T, Hutton M. Radiotherapy for acute, high-grade spinal cord compression caused by vertebral hemangioma. Spine J 2016;16(3):195-6. https://doi.org/10.1016/j.spinee.2015.10.018

28. Rades D, Bajrovic A, Alberti W, Rudat V. Is there a dose-effect relationship for the treatment of symptomatic vertebral hemangioma? Int J Radiat Oncol Biol Phys 2003;55(1):178-81. https://doi.org/10.1016/s0360-3016(02)03734-3

29. Kaoudi A, Capel C, Chenin L, Peltier J, Lefranc M. Robot-assisted radiofrequency ablation of a sacral s1-s2 aggressive hemangioma. World Neurosurg 2018;116:226-9. https://doi.org/10.1016/j.wneu.2018.05.060

30. Manning HJ. Symptomatic hemangioma of the spine. Radiology 1951;56(1):58-65. https://doi.org/10.1148/56.1.58 
31. Raco A, Ciappetta P, Artico M, Salvati M, Guidetti G, Guglielmi G. Vertebral hemangiomas with cord compression: the role of embolization in five cases. Surg Neurol 1990;34(3):164-8. https://doi.org/10.1016/0090-3019(90)90067-y

32. Grau SJ, Holtmannspoetter M, Seelos K, Tonn JC, Siefert A. Giant multilevel thoracic hemangioma with spinal cord compression in a patient with Klippel-Weber-Trenaunay syndrome: case report. Spine (Phila Pa 1976) 2009;34(14):E498-E500. https://doi.org/10.1097/BRS.0b013e3181a4e4b8

33. Wang GX, Mu YD, Che JY, Zhang GF, Jiang G, Gao CP. Compressive myelopathy and compression fracture of aggressive vertebral hemangioma after parturition: A case report and review of literature. Medicine (Baltimore) 2019;98(50):e18285. https://doi.org/10.1097/MD.0000000000018285 